\title{
Análisis Cinemático de la Masticación de una Granola Prototipo en Contraste con la Masticación de Maní
}

\author{
Kinematic Analysis of Chewing by Using a Granola Prototype and Peanut
}

\author{
Constanza Farfán¹,2; Joel Jerson Coaquira Quispe³; María Florencia Lezcano; \\ Alain Arias1; Pablo Navarro1 \& Ramón Fuentes ${ }^{1,4}$
}

FARFÁN, C.; COAQUIRA, J. J. Q.; LEZCANO, M. F.; ARIAS, A.; NAVARRO, P. \& FUENTES, R. Análisis cinemático de la masticación de una granola prototipo en contraste con la masticación de maní. Int. J. Odontostomat., 14(2):198-204, 2020.

RESUMEN: La masticación se ha estudiado desde diferentes puntos de vista, utilizando alimentos de prueba naturales y artificiales. La evidencia es escasa cuando se analizan alimentos a base de cereales, que van desde cereales para el desayuno hasta barras de granola. El investigar este tipo de alimentos, se vuelve importante para entender el comportamiento de la masticación frente a alimentos con diferentes composiciones y texturas, y como estas características pueden influir en el proceso masticatorio. Se analizó la masticación desde un punto de vista cinemático, en sujetos jóvenes dentados. El alimento de prueba utilizado fue granola prototipo y maní, este último se ha estudiado en sujetos con rehabilitación protésica y su consumo se recomienda en esta población. Se analizaron las características cinemáticas de la masticación como numero de ciclos, frecuencia masticatoria, velocidad de masticación de ascenso y descenso, y el área de masticación en los tres planos del espacio. Se relacionaron los movimientos masticatorios con los movimientos mandibulares bordeantes que conformaron el polígono de Posselt, este también se analizó en los tres planos espaciales. En todas las variables analizadas la granola presento valores mayores, excepto en el número de ciclos masticatorios, sólo se encontraron diferencias estadísticamente significativas $(p=0,03)$ al comparar la velocidad (ascenso y descenso) y el área de masticación en el plano horizontal.

PALABRAS CLAVE: cereales; maní; masticación; movimiento mandibular bordeante; características cinemáticas; artoculografía electromagética.

\section{INTRODUCCIÓN}

La masticación es el primer paso de la digestión, consiste en un conjunto de eventos del sistema estomatognático destinados a fragmentar los alimentos en partículas de un tamaño adecuado para su deglución (van der Bilt et al., 1994; Santos et al., 2006) y posterior procesamiento a través del sistema digestivo (van der Bilt et al., 1993). En los últimos años, se han estudiado los beneficios para la salud relacionados al proceso de masticación, investigando la importancia de variables como el tiempo de residencia de los alimentos en la boca, vinculándose, por ejemplo, con el control de la ingesta de alimentos (De Wijk et al., 2008; Forde et al., 2013a,b). Dado que la masticación no es un proceso estático y que hay una continua retroalimentación entre el sistema nervioso central y la cavidad oral para controlar la masticación hasta la deglución, surge el estudio de la dinámica de la masticación (Brown \& Braxton, 2000; Lillford, 2000), ante este escenario, toma relevancia el estudio de la mecánica masticatoria, que desde sus inicios ha intentado describir los movimientos mandibulares (MM) durante la masticación en base a la cinemática mandibular, donde la mayoría de los estudios disponibles en la literatura analizan los movimientos con equipos limitados al análisis en dos dimensiones (Toman et al., 2012, Kuninori et al., 2014), sin embargo actualmente existen instrumentos de alta precisión, que hacen posible el estudio de la cinemática masticatoria

\footnotetext{
${ }^{1}$ Centro de Investigación en Ciencias Odontológicas (CICO), Facultad de Odontología, Universidad de La Frontera, Temuco, Chile.

2 Universidad Adventista de Chile, Chillán, Chile.

${ }^{3}$ Facultad de Ingeniería y Arquitectura (FIA), Universidad Peruana Unión - Filial Juliaca (UPeU), Perú.

${ }^{4}$ Departamento de Odontología Integral Adultos, Facultad de Odontología, Universidad de La Frontera, Temuco, Chile.
} 
en los tres planos del espacio (Fuentes et al., 2018a,b). Farrell et al. (1956) demostraron que la capacidad de los alimentos para ser absorbidos en el sistema digestivo no solo dependía de la masticación sino también del tipo de alimento, y de sus propiedades físicas, como la fragilidad y resistencia a la fractura (Khan \& Vincent, 1993), también se ha estudiado la influencia de diferentes texturas, durezas y tamaño de la partícula en el proceso de masticación (Pröschel \& Hofmann, 1988; Jalabert-Malbos et al., 2007). De esta forma, se ha descrito que, con el aumento del tamaño de las partículas de alimento, se ve aumentado el tiempo de masticación (Grigoriadis et al., 2014), también lo hace la actividad electromiográfica de los músculos de la masticación (Miyawaki et al., 2000), la velocidad de desplazamiento de la mandíbula y el número de ciclos (M., C. and A., 2002). En cuanto a la dureza de los alimentos, se ha demostrado que también influye en la masticación, viéndose afectada principalmente la actividad muscular de los músculos masticatorios (Miyawaki et al.; Bhatka et al., 2004), mientras que la velocidad de desplazamiento de la mandíbula y el tiempo de masticación, no hay unaminidad en los diferentes estudios, esto puede deberse a diferencias en los protocolos de medición, instrumentos de medición o alimento de prueba utilizado (Foster et al., 2006; Grigoriadis et al.).

Dentro de los diferentes alimentos que se han utilizado como prueba para evaluar su comportamiento durante la masticación, se incluyen las almendras, zanahorias crudas y maní, como los alimentos naturales más utilizados que tienen la ventaja de permitir que los sujetos se sientan y mastiquen de forma natural (Yurkstas \& Manly, 1950; Kapur et al., 1964; Yoshida et al., 2009). Está establecido que el maní es un buen representante de los alimentos blandos y que la zanahoria cruda es buen representante de los alimentos duros de la dieta (Fueki et al., 2013). Ambos alimentos son baratos, uniformes y aceptados para consumo en humanos (Fueki et al.). Algunas personas con prótesis pueden masticar alimentos blandos como el maní de igual manera que las con dentición natural, pero fallan con alimentos más duros como la zanahoria (Fueki et al.). A pesar de la gran cantidad de estudios que relacionan la masticación con diferentes alimentos de prueba, no son suficientes cuando se consideran alimentos a base de cereales, que van desde cereales para el desayuno hasta barras de granola. El investigar este tipo de alimentos, se vuelve importante para entender el comportamiento de la masticación frente a alimentos de diferentes composiciones y texturas, y como estas características pueden influir en la masticación, más aún si consideramos que su consumo se ha vuelto muy frecuente en los últimos años (Grigoriadis et al.; Al-Omiri, 2018). Un estudio, llevado por Le Révérend et al. (2016), comparo las características cinemáticas de la masticación en sujetos jóvenes, al consumir diferentes productos comerciales a base de cereales. Los resultados arrojaron que la granola toma un tiempo significativamente mayor para ser masticado y también requiere un mayor número de ciclos masticatorios. Este producto fue considerado como un alimento recomendado para la masticación de adultos (Al-Omiri).

De acuerdo a lo anterior, el objetivo del presente trabajo es analizar, desde el punto de vista cinemático, la masticación de una granola prototipo y compararla con la masticación de maní, el cual es considerado representativo de los alimentos blandos y posible de masticar por portadores de prótesis (Fueki et al.).

\section{MATERIAL Y MÉTODO}

El siguiente análisis es sólo cinemático (movimiento) y está interpretado desde un punto de vista odontológico (función masticatoria).

Declaración de Ética. Se realizó un estudio observacional, de corte transversal que contó con la aprobación del Comité de Ética Científico de la Universidad de La Frontera ( $\mathrm{N}^{\circ}$ de protocolo 080_18). De conformidad con la Declaración de Helsinki de la Asociación Médica Mundial (2008), el consentimiento informado voluntario se obtuvo por escrito por los voluntarios antes de la participación, habiendo sido informados de la naturaleza del estudio.

Participantes. Se incluyeron en este análisis 7 participantes ( 3 hombres y 4 mujeres entre 19 y 28 años de edad; promedio 24,5 años). Se aplicaron los siguientes criterios para la selección de los participantes: Jóvenes mayores de 18 años, hombres y mujeres, sanos, sin tratamientos médicos actuales, con arco dentario maxilar y mandibular contínuo y completo (hasta el primer molar), con normoclusión y sin aparatos de ortodoncia. Se excluyeron aquellos que presentaban problemas articulares o musculares que impidieran realizar una correcta masticación y aquellos participantes alérgicos al maní o a algún componente de la granola como: avena, quinua, coco o canela. 
Alimento de prueba. Dos tipos de alimentos fueron utilizados para este estudio: Granola prototipo y Maní. A cada participante se le entrego 3,7 gramos de cada alimento, con la instrucción de masticar de forma natural sin indicar un lado de preferencia. Ambos alimentos fueron proporcionados en la misma cantidad de partículas, con tamaños similares de aproximadamente: $10 \mathrm{~mm}$ de diámetro y $15 \mathrm{~mm}$ de largo (Fig. 1).

Para la preparación de la granola prototipo se utilizó hojuelas de avena, maní triturado, kiwicha y quinua expandida, partículas de coco, ajonjolí, canela en polvo, aceite, azúcar, glucosa y sal. Los ingredientes secos fueron combinados en una mezcladora (AES-40/1 Braesi, Brasil) por un tiempo de 10 min hasta alcanzar una mezcla homogénea. Por otra parte, se preparó el jarabe a partir de azúcar, aceite, glucosa,

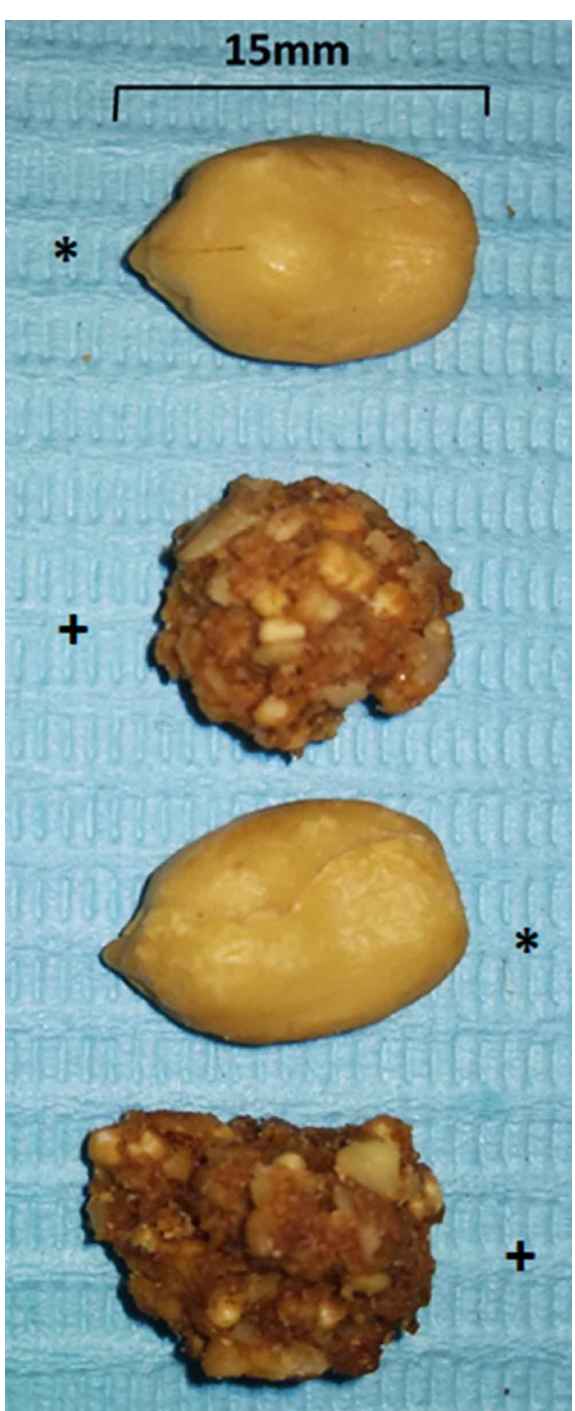

Fig. 1. Alimentos de prueba utilizados: $\left(^{*}\right)$ maní; (+) granola prototipo. sal y agua; el jarabe fue mezclado con los ingredientes secos por 10 min para la humectación homogénea de la mezcla, posteriormente fue coccionado a $150^{\circ} \mathrm{C}$ por 25 min en un horno convectivo (Peyron et al., 2000; Nova Industrial Tools, Perú), se almaceno en bolsas de polipropileno hasta su análisis químico y pruebas de masticación. El contenido de proteínas y humedad se analizaron de acuerdo con el método Kjeldahl y 925.45D (Association of Official Agricultural Chemists, 1990) (Le Révérend et al.); el contenido de grasa, ceniza, fibra cruda según la NTP (Association of Official Agricultural Chemists; NTP, 2001) y los hidratos de carbono se calculó por diferencia.

En la Tabla I se presenta la composición química de la granola prototipo, donde se puede apreciar un alto contenido en grasa, ello es debido a los ingredientes secos y líquidos: maní, coco y aceite. El contenido de humedad es característico para granolas comerciales (NTP 209.265. 2001). Aporta un cuarto ingesta en energía total que debe consumir una persona. Respecto al contenido de proteínas y cenizas es acorde a los presentado por Souza \& Silva (2015) y Granada et al. (2003).

Método de grabación. Se registraron los MM bordeantes en los tres planos del espacio y los movimientos masticatorios para 3,7 g de maní (Gonçalves et al., 2014) y para 3,7 g de granola (prototipo) mediante un articulógrafo electromagnético (EMA) 3D modelo AG501 (Carstens Medizinelektronik, Bovenden, Alemania). Para este estudio se utilizaron cuatro sensores del EMA 3D, que se fijaron a la piel del participante con un pegamento tisular biocompatible (Epiglu $囚$, Meyer Haake, Alemania). Estos sensores se distribuyeron en los siguientes puntos de la cabeza del participante: punto cutáneo del mastoide derecho $\left(1^{\circ}\right)$ e izquierdo $\left(2^{\circ}\right)$, glabela $\left(3^{\circ}\right)$ y línea mediana inter-incisiva mandibular $\left(4^{\circ}\right)$. Los tres primeros sensores se utilizaron como un sistema de referencia para normalizar el registro del sensor que se encuentra en la mandíbula y así desestimar el movimiento de la cabeza del participante. Los registros se realizaron según Fuentes et al. (2018a,b).

Se analizaron las características cinemáticas del movimiento: cantidad de ciclos masticatorios desarrollados hasta la primera deglución, la frecuencia masticatoria expresada en ciclos por minuto, la velocidad de descenso y de ascenso desde punto medio interincisal de la mandíbula, el área de los ciclos individualizados en el plano frontal, sagital y horizontal, la razón entre el área de cada ciclo y el área del polígono de Posselt en los planos frontal, sagital y horizontal.

La recolección de los datos se registró en una planilla Microsoft Office Excel, se realizó un análisis descriptivo de los datos para los que se determinó el promedio y su respectiva desviación estándar, se realizó la prueba no paramétrica de los signos para muestras relacionadas. Para el análisis de los datos se usó el programa estadístico IBM SPSS Statistics (versión 23.0). Un valor de $p<0,05$ fue elegido como umbral para significancia. 
Tabla I. Composición química de la granola. Componentes granola prototipo,
Humedad

Proteína*

Grasa

Ceniza

Fibra cruda

Carbohidratos

Energía total (Kcal)

${ }^{*} \mathrm{~N} \times 6,25$

\section{Componente}

$\mathrm{g} / 100 \mathrm{~g}$

$5,84 \pm 0,0571$

$10,23 \pm 0,014$

$23,03 \pm 0,049$

$1,63 \pm 0,007$

$6,25 \pm 0,064$

$53,04 \pm 0,049$

$460,29 \pm 0,304$
$433,44 \pm 193,75 \mathrm{~mm}^{2}$, para el plano sagital el área fue 282,11 $\pm 151,43 \mathrm{~mm}^{2}$, y para el plano horizontal el área fue $86,65 \pm 23,58$ $\mathrm{mm}^{2}$. Las áreas de los polígonos fueron analizadas en cuanto a su relación con las áreas de los ciclos masticatorios (Fig. 4), para maní (a) y granola (b), obteniéndose la razón de la siguiente manera: área_ciclo/área_polígono (Tabla III), esto para cada alimento de prueba. Se observó que los valores medios para la granola fueron mayores. Sin embargo, tampoco se encontraron diferencias estadísticamente significativas.

\section{MASTICACIÓNGRANOLA}

\section{RESULTADOS}

Se analizaron los MM bordeantes y masticatorios. La masticación de granola, en general presenta valores medios mayores que la masticación de maní en todas las variables analizadas excepto en el número de ciclos necesarios para triturar alimento (Tabla II). Sin embargo, sólo se encontraron diferencias estadísticamente significativas en la velocidad de masticación de ascenso y descenso $(p=$ 0,03 ) y en el área horizontal del ciclo masticatorio $(p=0,03)$. La Figura 2 muestra el comportamiento de la masticación de maní, y la Figura 3 de granola, indicando la tendencia de cada variable: Frecuencia masticatoria (ciclos/min); Área de masticación frontal, sagital y horizontal $\left(\mathrm{mm}^{2}\right)$; velocidad de masticación ascendente y descendente $(\mathrm{mm} / \mathrm{s})$.

Del estudio de los MM bordeantes, se obtuvo el polígono de Posselt y este fue analizado en las tres dimensiones del espacio. El promedio de las áreas registradas para el polígono de Posselt en el plano frontal fue

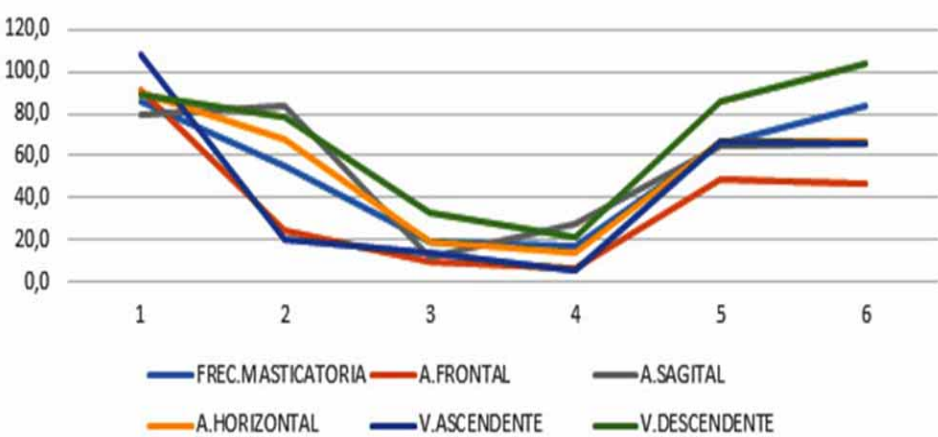

Fig. 2. Tendencia de variables: Frecuencia masticatoria (ciclos/min); Área de masticación frontal, sagital y horizontal $\left(\mathrm{mm}^{2}\right)$; Velocidad de masticación ascendente y descendente $(\mathrm{mm} / \mathrm{s})$, para la masticación de Maní.

\section{MASTICACIÓNGRANOLA}

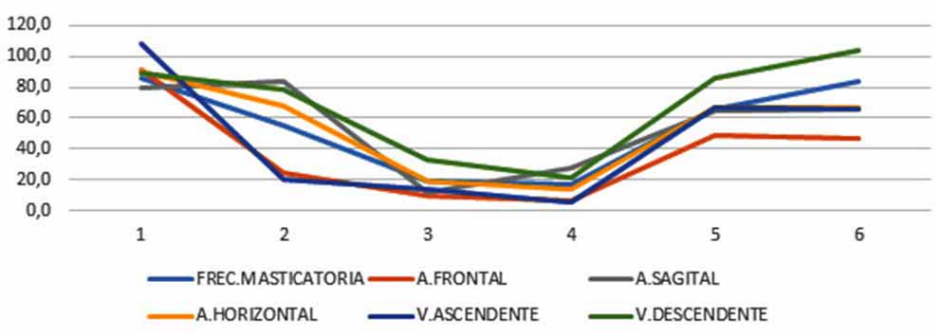

Fig. 3. Tendencia de variables: Frecuencia masticatoria (ciclos/min); Área de masticación frontal, sagital y horizontal $\left(\mathrm{mm}^{2}\right)$; velocidad de masticación ascendente y descendente $(\mathrm{mm} / \mathrm{s})$, para la masticación de Granola Prototipo.

Tabla II. Estadística descriptiva de las características cinemáticas de la masticación.

\begin{tabular}{lccccccc}
\hline $\begin{array}{l}\text { Alimento } \\
\text { de prueba }\end{array}$ & Ciclos & $\begin{array}{c}\text { Frecuencia } \\
\text { masticatoria } \\
\text { ciclos/min }\end{array}$ & $\begin{array}{c}\text { Área } \\
\left(\mathrm{mm}^{2} \pm \mathrm{DS}\right)\end{array}$ & $\begin{array}{c}\text { Área } \\
\text { Sagital } \\
\left(\mathrm{mm}^{2} \pm \mathrm{DS}\right)\end{array}$ & $\begin{array}{c}\text { Área } \\
\text { Horizontal } \\
\left(\mathrm{mm}^{2} \pm \mathrm{DS}\right)\end{array}$ & $\begin{array}{c}\text { Velocidad } \\
\text { Ascenso } \\
\mathrm{mm} / \mathrm{s}\end{array}$ & $\begin{array}{c}\text { Velocidad } \\
\text { Descenso } \\
\mathrm{mm} / \mathrm{s}\end{array}$ \\
\hline Maní & $27,5 \pm 11,8$ & $85,2 \pm 12,3$ & $39,9 \pm 19,4$ & $14,6 \pm 7,7$ & $9,1 \pm 5,3$ & $55,1, \pm 6,6$ & $55,9 \pm 10,3$ \\
Granola & $19,8 \pm 6,2$ & $90,7 \pm 9,7$ & $54,8 \pm 27,2$ & $17,6 \pm 8,5$ & $15,1 \pm 8,8$ & $66,3 \pm 11,8$ & $72,2 \pm 19,7$ \\
$\mathrm{p}=$ & 0,06 & 0,21 & 0,37 & 0,21 & $0,03^{*}$ & $0,03^{*}$ & $0,03^{*}$ \\
\hline
\end{tabular}

Tabla III. Razón entre movimientos mandibulares masticatorios y bordeantes.

\begin{tabular}{lccc}
\hline $\begin{array}{l}\text { Razón (área-ciclos/área-polígonos) } \\
\text { Alimento de prueba }\end{array}$ & Rate F & Rate S & Rate H \\
\hline Maní & $8 \pm 2 \%$ & $5 \pm 2 \%$ & $10 \pm 6 \%$ \\
Granola & $12 \pm 5 \%$ & $7 \pm 3 \%$ & $17 \pm 9 \%$ \\
$\mathrm{p}=$ & 0,9 & 0,9 & 0,9 \\
\hline
\end{tabular}



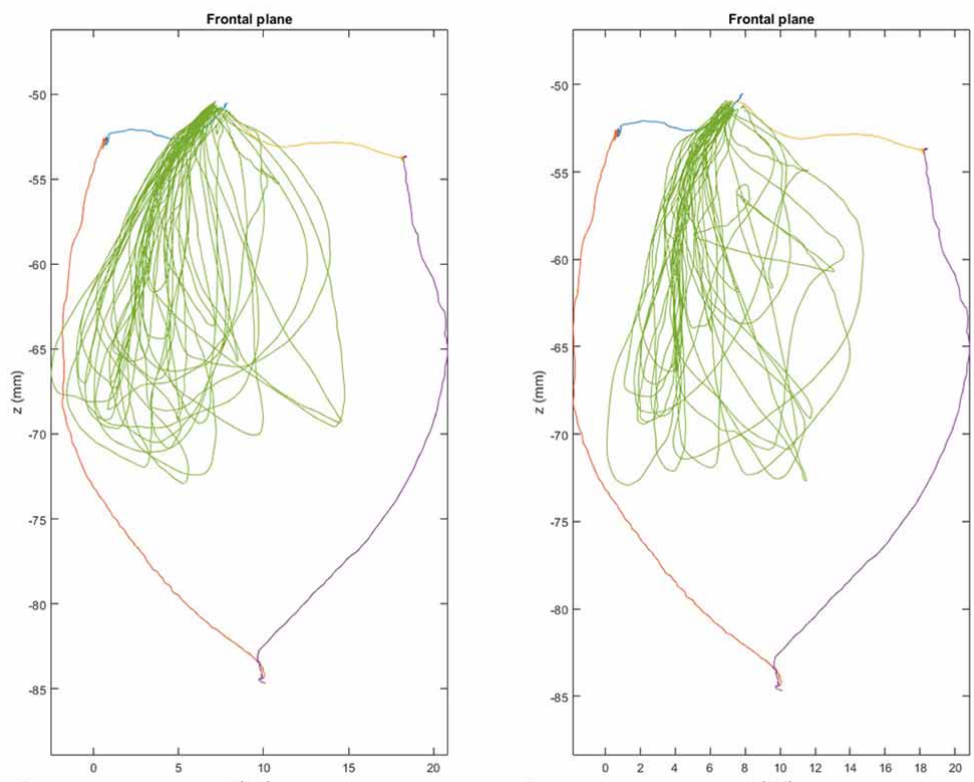

Fîg. 4. Representación de polígono de ${ }^{\mathrm{B}}$ Posselt en el plâno frontal, relacionado con los ciclos masticatorios del mismo sujeto, contraste entre alimento maní (A) y granola (B). consumir diferentes productos a base de cereales, dentro de los alimentos estudiados se encontró la granola. Los resultados de este estudio se relacionan con los nuestros, ya que, al comparar la granola con los otros alimentos de prueba, existe un aumento en la frecuencia, esta toma un mayor tiempo para ser masticada aumentando el número de ciclos masticatorios y actividad electromiográfica, se indica que la tasa promedio de actividad EMG por ciclo de masticación, es mayor al consumir granola, en comparación con otros alimentos a base de cereal (Al-Omiri). De los alimentos analizados por Le Révérend et al., la granola fue considerada como el producto más estimulante, esto por sus características cinemáticas masticatorias y por la actividad electromiográfica de sus músculos, por ende los autores recomiendan su consumo en adultos.

\section{DISCUSIÓN}

En este trabajo, se analiza la masticación de una granola prototipo desde el punto de vista cinemático y se compara con la masticación de maní (alimento de referencia). Al analizar las características cinemáticas que comparamos (Número de ciclos masticatorios, frecuencia de masticación, área de los ciclos masticatorios en plano frontal, sagital y horizontal, y la velocidad de ascenso y descenso mandibular), obtuvimos que el valor de todas las variables estudiadas fue mayor en la granola en comparación al maní, excepto en el número de ciclos masticatorios necesarios para triturar cada alimento. Sin embargo, solo existieron diferencias estadísticamente significativas en la velocidad de masticación (ascenso y descenso) y en el área de masticación horizontal $(p=0,03)$. Esto responde a la diferencia en la estructura de cada alimento utilizado, ya que el maní al ser un alimento compacto, en comparación a la granola, que se compone de trozos, es necesario aumentar el número de ciclos para la correcta trituración, y con esto disminuye la velocidad.

Según un reciente estudio realizado por Le Révérend et al., donde comparo el comportamiento de la masticación en sujetos jóvenes, según sus características cinemáticas y electromiográficas al
Si consideramos que con el paso del tiempo, dentro de las alteraciones que ocurren en el sistema estomatognático, la perdida de dientes es la más frecuente y que además de afectar la calidad de vida, produce alteraciones en la homeostasis del sistema estomatognático (Sáez Carriera et al., 2007), donde se ve afectada la función masticatoria y en la mayoría de los casos estos sujetos deben cambiar su alimentación, escogiendo alimentos blandos, fáciles de masticar, restándole importancia al valor nutricional (Tremayne \& Harrison, 2016). Hay evidencia suficiente sobre el impacto que la masticación tiene sobre la activación cerebral; estudios descriptivos en humanos muestran las diferentes áreas del cerebro que son activadas durante la masticación, tales como la corteza sensoriomotora primaria, suplementaria área motora, ínsula, cuerpo estriado, tálamo y cerebelo (Viggiano et al., 2015); este aumento del flujo cerebral influye en mejorar por ejemplo, procesos como el aprendizaje y memoria, pudiendo considerar que la función masticatoria actúa como factor protector en aquellos sujetos con deterioro cognitivo o enfermedades neurodegenerativas (Sesay et al., 2000). Yokoyama et al. (2017) correlaciona positivamente la comida moderadamente dura con el aumento del flujo cerebral. En nuestro estudio utilizamos maní y granola, el maní es considerado un alimento que puede ser masticado por portadores de prótesis (Fueki et al.), ante este escenario y teniendo en cuenta que la granola sólo mostro diferencias significativas con 
el maní, en la velocidad de masticación y en el área de masticación horizontal, lo que nos muestra un patrón de masticación con movimientos laterales y en sentido antero- posterior aumentado, podemos considerar a la granola como un alimento recomendado para ser masticado por adultos mayores con perdida dentaria, y que además produce una activación mayor del sistema masticatorio, presentando valores promedios más altos en todas las variables analizadas en comparación con el maní, aportando además un valor nutricional que muchas veces no se considera al momento de escoger un alimento que pueda ser masticado por un adulto con perdida dentaria.

A pesar de esto, consideramos que debe aumentarse el tamaño de la muestra para obtener resultados con mayor poder estadístico, y complementar el estudio de la cinemática masticatoria con por ejemplo electromiografía de superficie, se vuelve importante al momento de evaluar el impacto de la textura y dureza de diferentes alimentos, en la actividad sensorial de los músculos de la masticación. Realizar estos estudios en población rehabilitada con prótesis dental, ayudará a complementar los estudios actuales de la industria alimentaria, que buscan alimentos que cumplan funciones nutricionales específicas para esta población y que además el proceso de masticación de estos alimentos sea favorable para el deterioro neurodegenerativo producto de la edad (Sesay et al).

\section{CONCLUSIÓN}

Esta evidenciado que el maní es un alimento que puede ser masticado por portadores de prótesis. Nuestros resultados preliminares muestran que solo para la velocidad de masticación y el área de masticación horizontal fue significativamente mayor para granola, en comparación al maní. Además, no se halló evidencia que indique que la granola analizada no pueda ser masticada por portadores de prótesis, por lo tanto, recomendamos el consumo de granola en adultos mayores.

\section{AGRADECIMIENTOS}

Dr. Ramón Fuentes y su grupo de investigación han recibido el apoyo de la Oficina de Investigación, Universidad de La Frontera. Este trabajo es parte del proyecto DFP18-0037.
FARFÁN, C.; COAQUIRA, J. J. Q.; LEZCANO, M. F.; ARIAS, A.; NAVARRO, P. \& FUENTES, R. Kinematic analysis of chewing by using a granola prototype and peanut. Int. J. Odontostomat., 14(2):198-204, 2020.

ABSTRACT: Chewing has been studied from different points of view, using natural and artificial foods test. When analyzing cereal-based foods, from breakfast cereals to granola bars, the evidence is scarce. Investigate this type of food is important to understand the behavior of chewing, with foods of different compositions and textures, and how these characteristics can influence the chewing process. Chewing was analyzed from a cinematic point of view, in young subjects complete dental. The test food used was prototype granola and peanuts, last one has been studied in subjects with prosthetic rehabilitation and its consumption is recommended in this population. The kinematic characteristics of chewing were analyzed: number of cycles, chewing frequency, ascent and descent chewing speed, and the chewing area in the three planes of space. The masticatory movements were related to the bordering mandibular movements, that formed the Posselt polygon, which was also analyzed in the three spatial planes. In all the variables analyzed, granola showed higher values, except in the number of chewing cycles, only statistically significant differences $(p=0.03)$ were found when comparing speed (ascent and descent) and the chewing area in the horizontal plane.

PALABRAS CLAVE: cereals, peanuts, chewing, bordering jaw movement, kinematic characteristics, electromagnetic articulation.

\section{REFERENCIAS BIBLIOGRÁFICAS}

Al-Omiri, M. K. Muscle activity and masticatory efficiency with bilateral extension base removable partial dentures with different cusp angles. J. Prosthet. Dent., 119(3):369-76, 2018.

Association of Official Agricultural Chemists (AOAC). Official Methods of Analysis. $15^{\text {th }}$ ed. Rockville, Association of Official Agricultural Chemists, 1990.

Bhatka, R.; Throckmorton, G. S.; Wintergerst, A. M.; Hutchins, B. \& Buschang, P. H. Bolus size and unilateral chewing cycle kinematics. Arch. Oral Biol., 49(7):559-66, 2004.

Brown, W. E. \& Braxton, D. Dynamics of food breakdown during eating in relation to perceptions of texture and preference: a study on biscuits. Food Qual. Preference, 11(4):259-67, 2000.

de Wijk, R. A.; Zijlstra, N.; Mars, M.; de Graaf, C. \& Prinz, J. F. The effects of food viscosity on bite size, bite effort and food intake. Physiol. Behav., 95(3):527-32, 2008.

Farrell, J. H. The effect of mastication on the digestion of food. $\mathrm{Br}$. Dent. J., 100:149-55, 1956.

Forde, C. G.; van Kuijk, N.; Thaler, T.; de Graaf, C. \& Martin, N. Oral processing characteristics of solid savoury meal components, and relationship with food composition, sensory attributes and expected satiation. Appetite, 60(1):208-19, 2013.

Forde, C. G.; van Kuijk, N.; Thaler, T.; de Graaf, C. \& Martin, N. Texture and savoury taste influences on food intake in a realistic hot lunch time meal. Appetite, 60(1):180-6, 2013. 
Foster, K. D.; Woda, A. \& Peyron, M. A. Effect of texture of plastic and elastic model foods on the parameters of mastication. $J$. Neurophysiol., 95(6):3469-79, 2006.

Fueki, K.; Yoshida, E. \& Igarashi, Y. Association between occlusal curvature and food comminution and mixing in human young adults with permanent dentitions. Arch. Oral Biol., 58(4):377-83, 2013.

Fuentes, R.; Arias, A.; Lezcano, M. F.; Saravia, D.; Kuramochi, G.; Navarro, P. \& Dias, F. J. A new tridimensional insight into geometric and kinematic characteristics of masticatory cycles in participants with normal occlusion. BioMed Res. Int., 2018:2527463, 2018.

Fuentes, R.; Dias, F.; Álvarez, G.; Lezcano, M. F.; Farfán, C.; Astete, N. \& Arias, A. Application of 3D electromagnetic articulography in dentistry: mastication and deglutition analysis. Protocol report. Int. J. Odontostomat., 12(1):105-12, 2018.

Gonçalves, T. M. S. V.; Vilanova, L. S. R.; Gonçalves, L. M. \& Rodrigues Garcia, R. C. M. Effect of complete and partial removable dentures on chewing movements. J. Oral Rehabil., 41(3):177-83, 2014.

Granada, G.; Rosa, V.; Zambiazi, R. \& Koetz, P. Caracterização de granolas comerciais. Ciênc. Tecnol. Aliment., 23(1):87-91, 2003.

Grigoriadis, A.; Johansson, R. S. \& Trulsson, M. Temporal profile and amplitude of human masseter muscle activity is adapted to food properties during individual chewing cycles. J. Oral Rehabil., 41(5):367-73, 2014.

Jalabert-Malbos, M. L.; Mishellany-Dutour, A.; Woda, A. \& Peyron, M. A. Particle size distribution in the food bolus after mastication of natural foods. Food Qual. Preference, 18(5):803-12, 2007.

Kapur, K.; Soman, S. \& Yurkstas, A. Test foods for measuring masticatory performance of denture wearers. J. Prosthet. Dent., 14(3):483-91, 1964

Khan, A. A. \& Vincent, J. F. V. Anisotropy in the fracture properties of apple flesh as investigated by crack-opening tests. J. Mater. Sci., 28:45-51, 1993.

Kuninori, T.; Tomonari, H.; Uehara, S.; Kitashima, F.; Yagi, T. \& Miyawaki, $\mathrm{S}$. Influence of maximum bite force on jaw movement during gummy jelly mastication. J. Oral Rehabil., 41(5):338-45, 2014.

Le Révérend, B.; Saucy, F.; Moser, M. \& Loret, C. Adaptation of mastication mechanics and eating behaviour to small differences in food texture. Physiol. Behav., 165:136-45, 2016.

Lillford, P. J. The materials science of eating and food breakdown. MRS Bull., 25(12):38-43, 2000.

Miyawaki, S.; Ohkochi, N.; Kawakami, T. \& Sugimura, M. Effect of food size on the movement of the mandibular first molars and condyles during deliberate unilateral mastication in humans. $J$. Dent. Res., 79(7):1525-31, 2000.

NTP 209.265. Alimentos cocidos de reconstitución instantánea. Papilla. Enriquecido lácteo. Determinación de cenizas. Método gravimétrico. 2001.

Peyron, M. A.; Lassauzay, C. \& Woda, A. Effects of increased hardness on jaw movement and muscle activity during chewing of visco-elastic model foods. Exp. Brain Res., 142(1):41-51, 2000.

Pröschel, P. \& Hofmann, M. Frontal Chewing patterns of the incisor point and their dependence on resistance of food and type of occlusion. J. Prosthet. Dent., 59(5):617-24, 1988.

Sáez Carriera, R.; Carmona, M.; Jiménez Quintana, Z. \& Alfaro, X. Cambios bucales en el adulto mayor. Rev. Cuba. Estomatol., 44(4), 2007

Santos, C. E.; de Freitas, O.; Spadaro, A. C. C. \& Mestriner-Junior, W. Development of a colorimetric system for evaluation of the masticatory efficiency. Braz. Dent. J., 17(2):95-9, 2006.

Sesay, M.; Tanaka, A.; Ueno, Y.; Lecaroz, P. \& De Beaufort, D. G. Assessment of regional cerebral blood flow by xenon-enhanced computed tomography during mastication in humans. Keio $\mathrm{J}$. Med., 49 Suppl. 1:A125-8, 2000.
Souza, P. L. C. \& Silva, M. R. Quality of granola prepared with dried caju-do-cerrado (Anacardium othonianum rizz) and baru almonds (Dipteryx alata vog). J. Food Sci. Technol., 52(3):1712-7, 2015.

Toman, M.; Toksavul, S.; Saracoglu A.; Cura, C. \& Hatipoglu, A. Masticatory performance and mandibular movement patterns of patients with natural dentitions, complete dentures, and implantsupported overdentures. Int. J. Prosthodont., 25(2):135-7, 2012.

Tremayne, P. \& Harrison, P. Gastrointestinal care for older people. Nurs. Stand., 30(45):53-63, 2016.

van der Bilt, A.; Olthoff, L. W.; Bosman, F. \& Oosterhaven, S. P. Chewing performance before and after rehabilitation of postcanine teeth in man. J. Dent. Res., 73(11):1677-83, 1994.

van der Bilt, A.; Olthoff, L. W.; Bosman, F. \& Oosterhaven, S. P. The effect of missing postcanine teeth on chewing performance in man. Arch. Oral Biol., 38(5):423-9,1993.

Viggiano, A.; Manara, R.; Conforti, R.; Paccone, A.; Secondulfo, C.; Lorusso, L.; Sbordone, L.; Di Salle, F.; Monda, M.; Tedeschi, G.; et al. Mastication induces long-term increases in blood perfusion of the trigeminal principal nucleus. Neuroscience, 311:75-80, 2015.

Yokoyama, T.; Sato, M.; Natsui, S.; Kuboyama, N.; Suzuki, K.; Inaba, $H$. \& Shibuya, K. Effect of gum chewing frequency on oxygenation of the prefrontal cortex. Percept Mot. Skills, 124(1):58-71, 2017.

Yoshida, T.; Ishikawa, H.; Yoshida, N. \& Hisanaga, Y. Analysis of masseter muscle oxygenation and mandibular movement during experimental gum chewing with different hardness. Acta Odontol. Scand., 67(2):113-21, 2009.

Yurkstas, A. \& Manly, R. S. Value of different test foods in estimating masticatory ability. J. Appl. Physiol., 3(1):45-53, 1950.

Dirección para correspondencia:

Prof. Dr. Ramón Fuentes Fernández

Centro de Investigación en Ciencias Odontológicas

Facultad de Odontología

Universidad de La Frontera

Av. Francisco Salazar 1145

Temuco

CHILE

E-mail: ramon.fuentes@ufrontera.cl

Recibido : 11-11-2019

Aceptado: 24-12-2019 